\title{
Novas perspectivas na avaliação da função pulmonar de lactentes
}

\section{New perspectives in the evaluation of lung function in infants}

Fernanda de Cordoba Lanza, Ft., D.Sc.*, Gustavo Falbo Wandalsen, D.Sc.**, Carolina Lopes da Cruz, Ft. ${ }^{* * *}$, Dirceu Solé****

*Universidade Federal de São Paulo (UNIFESP/EPM) São Paulo/SP, ** Médico, Universidade Federal de São Paulo (UNIFESP/

EPM) São Paulo/SP, ***Especialista em Fisioterapia Pediátrica pela Universidade Federal de São Paulo (UNIFESP/EPM) São Paulo/SP, ****Professor Titular da Disciplina de Alergia, Imunologia Clínica e Reumatologia, Departamento de Pediatria, UNIFESPI

EPM, São Paulo/SP

\section{Resumo}

Objetivo: Realizar levantamento da literatura científica sobre provas de função pulmonar (PFP) em lactentes, suas aplicaçóes e perspectivas. Métodos: Foi realizada busca de artigos científicos (língua inglesa, portuguesa e espanhola) nas bases de dados: PubMed, Embase, Scielo e Lilacs. Palavras de busca utilizadas: lactente, prova de função pulmonar, volume corrente, fluxos expiratórios forçados, pletismografia e mecânica respiratória; de forma combinada ou isolada, entre os anos de 1940 a 2008 incluindo artigos de revisão, ensaios clínicos, consensos e livros. Resultados: Nos últimos anos houve grande avanço no aperfeiçoamento e padronização das PFP em lactentes. As técnicas mais empregadas são: análise dos parâmetros do volume corrente; mecânica passiva do sistema respiratório; análise dos volumes pulmonares por pletismografia ou por métodos de difusão; compressão toracoabdominal para obtenção de fluxos expiratórios forçados seja em volume corrente ou em volume pulmonar aumentado. Na maioria dos casos sedação é necessária para a realização desses testes e os derivados do Cloral são os sedativos mais empregados. As principais contraindicaçôes das PFP em lactentes são decorrentes da sedação: obstrução de via aérea, cardiopatias arrítmicas e neuropatias. Complicaçóes decorrentes desses exames são extremamente raras, mas equipe experiente e suporte para reanimação cardiorrespiratória são recomendados. Apesar de utilizadas principalmente em pesquisas, as PFP em lactentes já apresentam indicaçóes clínicas definidas. Conclusão: PFP pode ser considerada como ferramenta útil e viável no manejo e pesquisa de pneumopatias de lactentes. O conhecimento desses exames pode auxiliar diversos profissionais envolvidos nos cuidados desses pacientes aprimorando a terapêutica e trazendo novas perspectivas na avaliaçáo de terapias respiratórias.

Palavras-chave: prova de função pulmonar, lactente, pletismografia, fluxos expiratórios forçado.

\begin{abstract}
Objective: To review literature regarding lung function tests (LFT) in infants, their application and perspectives. Methods: English, Spanish and Portuguese articles were included, from PubMed, Embase, Scielo and Lilacs bases, dating from 1940 to 2008. Books, review articles, clinical trials and consensus were selected employing the following key-words: infant, lung function, tidal volume, forced expiratory flows, plethismography and respiratory mechanics. Results: Over the last years there has been a marked improvement in the development and standardization of LFT in infants. The most employed techniques are: tidal breathing parameters analysis, passive respiratory mechanics, lung volumes (plethismography or diffusion methods), forced expiratory flows (thoracoabdominal compression) either from tidal volume or from raised volume. Sedation is necessary in the majority of tests and Choral derivatives are most commonly used. Main contraindications of LFT in infants are upper airway obstruction, and heart and neurological diseases. Complications of the tests are extremely rare but trained personnel and available resuscitation equipment is recommended. Although mainly employed for research, LFT in infants already have defined clinical indications. Conclusion: LFT in infants can actually be considered a useful and feasible tool in the management and research of infants with lung diseases. The knowledge of LFT's can be helpful for health professionals involved in infant care and for new perspectives in the evolution of respiratory therapy.
\end{abstract}

Key-words: lung function test, infant, plethysmography, forced expiratory flow. 


\section{Introdução}

O crescimento pulmonar adequado nos primeiros anos de vida é de particular importância especialmente porque o término da alveolizaçáo, iniciado no terceiro trimestre de gestação, finaliza por volta dos oito anos de idade [1]. Vários fatores tornam o lactente mais susceptível a afecções respiratórias tais como: sistema imunológico em desenvolvimento; caixa torácica e pulmóes em crescimento e músculos respiratórios não estruturados [2]. De fato, as infecções respiratórias em lactentes contribuem para o aumento a procura por serviço médico, e incremento no número de hospitalizaçóes e de óbitos. Estima-se que entre 40 e $60 \%$ das consultas médicas realizadas em lactentes sejam por queixas respiratórias e que praticamente todos eles tenham sido acometidos pelo menos uma vez por infecçôes do sistema respiratório. Dados internacionais indicam que cerca de $40 \%$ dos lactentes apresentarão crise de sibilância nos primeiros três anos de vida [3,4]. Há evidências, entretanto, que no Brasil essa prevalência seja bem maior [5,6].

$\mathrm{O}$ avanço nos cuidados peri e neonatais tem contribuído com o aumento na sobrevida de crianças cada vez mais prematuras, consequentemente o número de crianças com doenças respiratórias crônicas tem aumentado prejudicando o crescimento do sistema respiratório. A exposição a produtos tóxicos do tabaco, poluição ambiental e determinadas condiçôes de moradia também podem comprometer o desenvolvimento pulmonar $[7,8]$.

A avaliação da função pulmonar é ferramenta essencial para compreensáo da fisiologia pulmonar de lactentes e adultos. Em lactentes, limitaçôes técnicas como a não resposta aos comandos verbais dificultavam sua realização. Nos últimos anos, o desenvolvimento de novas técnicas e o aperfeiçoamento das existentes possibilitaram grande avanço nessa área, gerando novas e promissoras perspectivas no estudo da função pulmonar desses indivíduos.

A prova de função pulmonar (PFP) em lactentes tem importante papel em diversas áreas de pesquisas e passou a ter aplicaçáo efetiva no diagnóstico e manejo das doenças pulmonares prevalentes nessa faixa etária e em estudos epidemiológicos para acompanhamentos de crianças com doenças respiratórias. Desta forma, torna-se necessário que os profissionais de saúde conheçam as diferentes formas de avaliar a função pulmonar, suas aplicaçôes, limitaçôes e perspectivas.

\section{Histórico}

A avaliação da função pulmonar em lactentes sempre foi limitada pela necessidade de cooperação para realização deste exame. Inicialmente, os testes em crianças avaliavam a complacência e a resistência pulmonar com equipamentos invasivos como os balóes esofágicos $[9,10]$. Em meados dos anos 60, iniciaram-se os estudos de fisiologia pulmonar em recém-nascidos e, em 1978, foi descrito pela primeira vez a avaliaçáo de curvas expiratórias forçadas em lactentes [9].
As avaliaçóes do volume corrente e a pletismografia foram inicialmente realizados no hospital Great Ormond Street, Inglaterra, após adaptaçóes feitas nos ventiladores mecânicos de pressão negativa utilizados durante epidemia de poliomielite [10]. Entretanto, apenas nos últimos 25 anos é que houve real incremento destas técnicas com a possibilidade de maior aplicabilidade prática [11].

$\mathrm{O}$ estudo da função pulmonar em lactentes permitiu a compreensão de mecanismos fisiológicos e patológicos importantes. Tais avaliaçóes contribuíram, por exemplo, para o entendimento das diferenças fisiológicas existentes entre os sexos, do crescimento dissináptico do pulmão, dos diferentes fenótipos de lactentes sibilantes, dos fatores de risco para asma e dos efeitos deletérios da exposiçáo ao tabaco no desenvolvimento pulmonar [11-14].

Ferramenta primordial para a realização da PFP é o transdutor dos sinais físicos através do qual a mensuração de todas as variáveis é feita. Existem vários tipos de transdutores que avaliam diferentes parâmetros fisiológicos. Na PFP, o transdutor utilizado é o pneumotacógrafo que mensura fluxo, e integrado no tempo define volumes.

\section{Prova de função pulmonar}

A prova de função pulmonar é a mensuração de diversas variáveis do sistema respiratório. Estas podem ser feitas separadamente ou em conjunto, dependendo do equipamento que se tem disponível. As diferentes variáveis mensuradas são descritas a seguir.

\section{Volume corrente}

A avaliação do volume corrente (VC) é uma das técnicas mais antigas e simples de medida da função pulmonar. Diferentes parâmetros podem ser obtidos a partir da análise do VC, tais como: tempo inspiratório, tempo expiratório, tempo total respiratório, tempo para alcançar o pico de fluxo expiratório $\left(\mathrm{t}_{\mathrm{PTEF}}\right)$, a frequência respiratória (Figura 1). É recomendado o registro dos movimentos respiratórios espontâneos por pelo menos 30 segundos, em diferentes mediçóes. As avaliaçóes com a utilização de máscara facial acoplada a um pneumotacógrafo são as mais empregadas e padronizadas na atualidade, podendo ser realizado com ou sem sedação do lactente [15].

A frequência respiratória é determinada de acordo com a interação entre as propriedades passivas e componentes ativos do sistema respiratório. As alteraçôes do tempo inspiratório, volume e fluxo observados no volume corrente, podem refletir distúrbio do controle da respiração ou do sistema respiratório $[16,17]$.

O mecanismo de controle do tempo inspiratório sofre influências da mecânica respiratória e da sobrecarga aplicada. Em sobrecargas resistivas o tempo mecânico é maior, enquanto em sobrecargas elásticas o tempo neural se alonga e, em ambas, há redução do volume inspirado e prolongamento do tempo ins- 
piratório. Doenças que cursam com hipoxemia e hipercapnia, também alteram o comando do centro respiratório e a contraçáo diafragmática, alterando a frequência respiratória [18].

Figura 1 - Diferentes formas de expressar as curvas de volume corrente e variáveis delas derivadas.

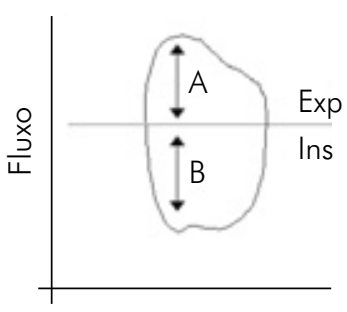

Volume

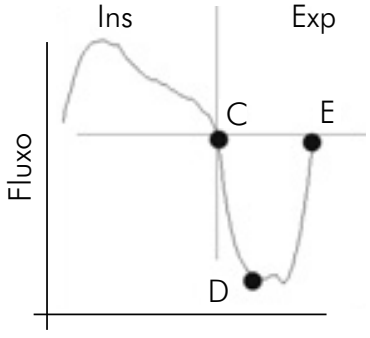

Tempo
A: pico de fluxo expiratório; $B$ : pico de fluxo inspiratório; C-D: tempo para alcançar o pico de fluxo expiratório $\left(t_{\text {PTEF }}\right)$; C-E: tempo expiratório ${ }_{(\mathrm{E})} ; \mathrm{CD} /$ $C E$ : relação $t_{P T E F} /_{t E^{\prime}} ; V C$ (volume corrente): inspiração + expiração.

O tempo expiratório pode ser modificado por obstrução laríngea ou por contração dos músculos abdominais. Nas sobrecargas elásticas há redução rápida do volume pulmonar, e nas resistivas o volume exalado é retardado. Ambos influenciarão o início da próxima inspiração, sendo esta, mais ou menos precoce [19].

A relação entre o $\mathrm{t}_{\mathrm{PTEF}} \mathrm{e}$ o tempo expiratório $\left(\mathrm{t}_{\mathrm{PTEF}} /_{\mathrm{tE}}\right)$ é um dos parâmetros mais estudados nessa técnica. Ele é dependente da atividade muscular, da retração elástica e da resistência da via aérea [20]. Em adultos, crianças e lactentes, com obstrução das vias aéreas o $\mathrm{t}_{\mathrm{PTEF}} /{ }_{\mathrm{tE}}$ está reduzido e associado à redução de fluxos pulmonares. Também está reduzido em lactentes que desenvolverão doenças do trato respiratório inferior com sibilância, e em filhos de mães tabagistas mostrando alteração precoce do sistema respiratório [21-23].

A simples análise do formato da curva do VC também pode trazer informaçóes úteis como a suspeita de obstrução fixa da via aérea, alteração de complacência pulmonar ou limitação grave ao fluxo expiratório [10,15]. O interesse por essa técnica permanece até os dias atuais devido a sua simplicidade, baixo custo e possibilidade de realizaçáo na ausência de sedação.

\section{Mecânica respiratória passiva}

As medidas de mecânica respiratória passiva visam avaliar o sistema respiratório (pulmão e caixa torácica) na ausência de atividade muscular, mensurando a resistência (Rsr), a complacência (Csr) e a constante de tempo do sistema respiratório $(\pi \mathrm{sr})$ [22]. A principal forma de se obter medidas de mecânica respiratória passiva é pelo método de oclusão da via aérea, única ou múltipla.

Para as medidas destes valores considera-se o sistema respiratório um modelo unicompartimental e linear, definindo-o como um único elemento. O relaxamento completo dos mús- culos respiratórios em lactentes, necessário para a avaliaçáo da mecânica passiva, é conseguido pela indução do reflexo de Hering Breuer (RHB), desencadeado pela rápida obstrução da via aérea (um segundo) ao início de uma expiração corrente [15]. A indução desse reflexo é obtida com maior sucesso durante os primeiros dois anos de vida [24]. Com a indução desse reflexo, grande parte da expiração é realizada na ausência de atividade muscular, evidenciada por um segmento linear na alça expiratória. A extrapolação do segmento linear da curva expiratória é utilizada para se estimar a constante de tempo, definida como o tempo necessário para o esvaziamento pulmonar. Deste modo, é possível o cálculo de outros parâmetros como a Rsr e a Csr [25,26] (Figura 2).

Figura 2 - Gráfico representativo da curva expiratória obtida na avaliação da mecânica passiva do sistema respiratório pela técnica de oclusão única.

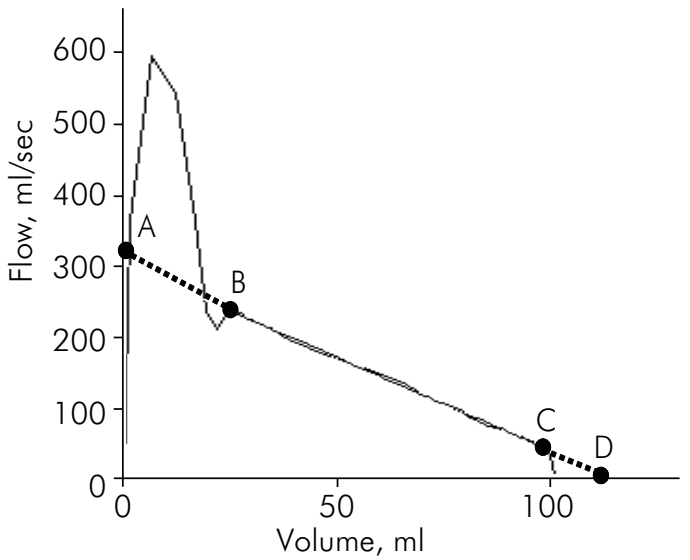

Segmento A-D: extrapolação para cálculo da constante de tempo do sistema respiratório; segmento B-C: porção linear da curva que significa relaxamento dos músculos respiratórios na fase expiratória da respiração.

Essa técnica assume que durante a oclusão da via aérea há equilíbrio das pressões do sistema respiratório e assim, a medida da pressão de boca refletiria a pressão de retração elástica do pulmão. Isto apenas será verdadeiro se houver relaxamento completo dos músculos respiratórios durante a oclusão. É recomendado, então, que se aceite apenas curvas em que a porção linear na curva de fluxo-volume seja observada em pelo menos $60 \%$ desta, que indica a ausência de atividade muscular espontânea $[27,28]$.

Essa técnica além de obter medidas reprodutíveis, tem sido utilizada em pesquisas de diversas doenças, no seguimento longitudinal da função pulmonar de lactentes sadios, assim como na avaliação de intervenções terapêuticas como de agentes broncodilatadores, corticosteróides inalados, surfactante exógeno e técnicas de fisioterapia respiratória [25,29-34].

\section{Volumes pulmonares}

Assim como nos adultos, os volumes pulmonares podem ser mensurados basicamente pelos métodos pletismográficos 
e pelos métodos de difusáo. Em lactentes, a pletismografia de corpo total é o método mais empregado e estudado até o momento. Nesta avaliação, o lactente é colocado dentro de uma caixa hermeticamente fechada (pletismógrafo), de volumes conhecidos, dotados de sensores internos, sensíveis a pequenas variaçôes de pressão. Ao final de uma inspiração corrente a via aérea é ocluída (geralmente entre 6 e 8 segundos) e, desta forma, o lactente realiza esforços respiratórios sem que haja fluxo de ar para dentro ou fora dos pulmóes. Assim, as variaçôes da pressão na via aérea são correlacionadas com as variaçôes de pressão dentro da caixa e é possível calcular o volume pulmonar no momento da oclusão. A capacidade residual funcional (CRF) é a medida mais utilizada nessa técnica e é definida como o volume pulmonar existente após uma expiração corrente, de outra forma, pela soma do volume residual (VR) e do volume de reserva expiratória (VRE). Para que os cálculos dos volumes pulmonares sejam precisos é necessário que o escape de ar pelo pletismógrafo seja conhecido, e que não haja fuga de ar durante os movimentos respiratórios [35-37].

A determinação dos valores de CRF já demonstrou ser útil em pesquisas e no manejo de lactentes com doenças respiratórias. De modo geral, valores reduzidos de CRF podem ser encontrados em lactentes com diminuição de complacência pulmonar e distúrbios de caixa torácica. Valores elevados são sugestivos de aprisionamentos aéreos, encontrados em doenças obstrutivas como a asma $[10,38]$. Valores de normalidade de CRF em função da idade e estatura são disponíveis na literatura e contribuem para a interpretação do exame [39-41.

Além da medida dos volumes pulmonares, diversos pletismógrafos são capazes de mensurar a resistência de vias aéreas (Rva). A Rva tem como vantagem ser medida durante o ciclo respiratório, e ser considerada a partir da avaliação dinâmica do sistema. Os valores de Rva e resistência pulmonar tendem a ser próximo em lactentes, pois o componente tecidual contribui modestamente à resistência pulmonar por ter pequena quantidade de colágeno e elastina. A técnica apropriada para a mensuração da Rva, entretanto, necessita que o lactente respire em bolsas aquecidas, sob condiçóes de pressão e umidade conhecidas. Isso dificulta sua reutilização e limita a aplicação prática. Novos métodos de compensação eletrônica já foram desenvolvidos, mas não estão devidamente padronizados até o momento.

\section{Fluxos forçados}

As curvas de fluxo-volume foram obtidas pela primeira vez, em lactentes, em 1978, após o desenvolvimento da técnica de compressão toracoabdominal [9]. Essa técnica inicial foi rapidamente aprimorada com o desenvolvimento de uma jaqueta inflável e, desde entấo, consiste na forma mais utilizada para se estudar a expiração forçada de lactentes (Figura 3). Há duas técnicas distintas, a que analisa curvas obtidas a partir do volume corrente (parcial) e a que analisa curvas obtidas de volume pulmonar elevado (RVRTC) [40].
Figura 3 - Esquema representativo do equipamento utilizado para realizar manobras de compressão toracoabdominal rápida.

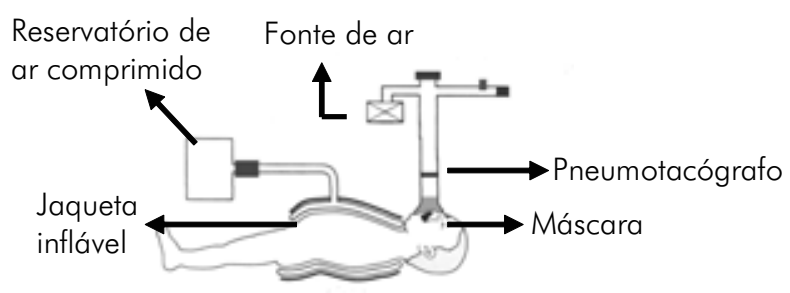

Nessas duas técnicas, uma jaqueta inflável, conectada a um grande reservatório de ar comprimido, é colocada em torno do tórax e abdômen do lactente. Esse respira espontaneamente conectado a um pneumotacógrafo e a uma máscara facial (figura 3). Quando o reservatório é liberado, a jaqueta é rapidamente inflada e uma curva expiratória forçada pode ser registrada pelo pneumotacógrafo. Curvas subsequentes são realizadas com pressão crescente de compressão da jaqueta até que não haja mais incremento nos fluxos e volumes expiratórios forçados (limitação de fluxo) [10,38].

$\mathrm{Na}$ técnica de expiração forçada parcial (PRTC), a compressão toracoabdominal é realizada após a inspiração normal, em volume corrente (Figura 4). Nesta técnica, o principal parâmetro analisado é o fluxo expiratório máximo no nível da CRF (V'maxCRF). Essa técnica foi amplamente utilizada. Inúmeros estudos de função pulmonar em lactentes já foram publicados empregando essa técnica, que é responsável por grande parte do conhecimento acumulado nesta área. Equaçôes para cálculo de valores de normalidade obtidos por essa técnica em lactentes sadios estão disponíveis [41,42]. A técnica de PRTC apresenta, porém, algumas limitações. A análise de parâmetros é limitada à faixa do VC e seu principal parâmetro não é muito reprodutível, uma vez que o volume em que a compressão toracoabdominal é efetuada (CRF) não é estável. Há ainda, dúvidas de que seja possível alcançar a limitação de fluxo em lactentes sem obstrução brônquica [10].

Figura 4 - Curva expiratória forçada parcial.

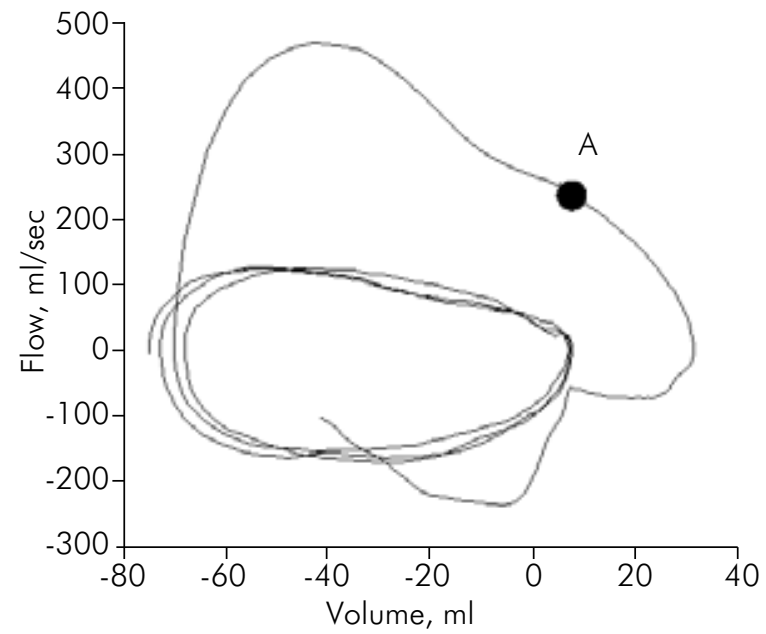

A: Fluxo expiratório forçado no nível da capacidade residual funcional (VmaxCRF) 
Recentemente a técnica PRTC foi aperfeiçoada, para tanto, fonte de ar comprimido e sistema de válvulas que permitem a administração de pressão positiva ao início da inspiração espontânea do lactente foram adicionados ao sistema. Tal pressão, previamente determinada $\left(20\right.$ ou $\left.30 \mathrm{cmH}_{2} \mathrm{O}\right)$, aumenta a capacidade inspiratória da criança, simulando a inspiração forçada que se aproxima da capacidade pulmonar total (Figura 5). A repetição deste procedimento é capaz de induzir o RHB na maioria dos lactentes e permitir, assim, que a compressão toracoabdominal seja efetuada até o volume residual, sem interferência da inspiração espontânea do lactente [42-45]. A curva expiratória obtida é muito semelhante a espirometria de crianças maiores e adultos, e permite o cálculo das mesmas variáveis: capacidade vital forçada (CVF), o volume expiratório forçado em distintos tempos $\left(\mathrm{VEF}_{0,5} \mathrm{eVEF}_{0,75}\right)$ e os fluxos expiratórios em diversas porçóes da CVF $\left(\mathrm{FEF}_{50}\right.$; $\mathrm{FEF}_{75} ; \mathrm{FEF}_{25-75}$. Essa nova técnica denominada de compressão torácica rápida em volume pulmonar elevado (RVRTC) demonstrou ser menos variável que sua antecessora [46-47] e ser suficientemente sensível para detectar obstrução brônquica em lactentes assintomáticos, além de discriminar lactentes com distintos graus de distúrbios respiratórios [10,12,48-50].

Figura 5 - Curva expiratória forçada com volume pulmonar elevado.

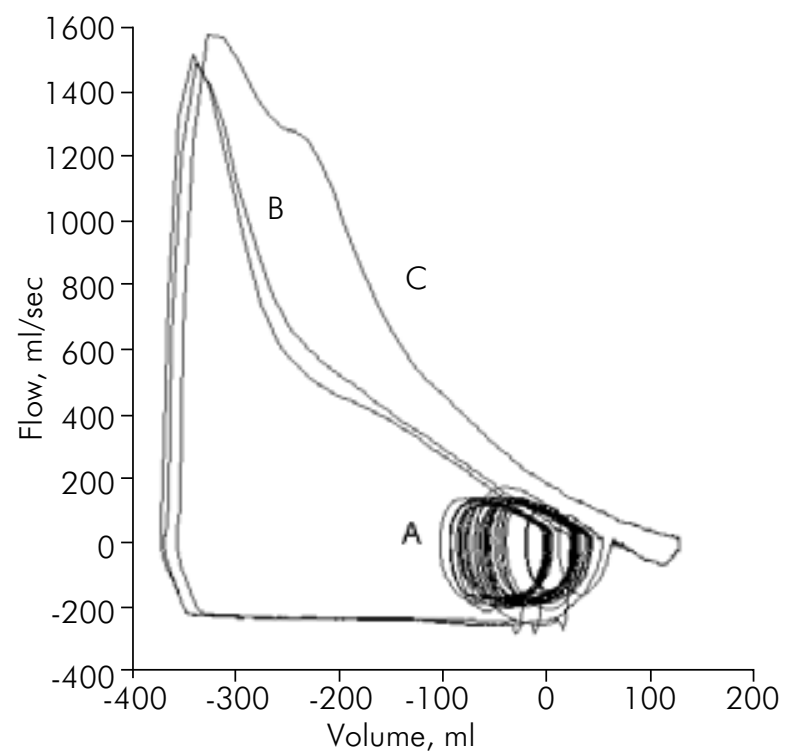

A: volume corrente; $B$ : curvas expiratórias passivas após inflação a 30 $\mathrm{cmH}_{2} \mathrm{O}$; $\mathrm{C}$ : curva expiratória forcada após a compressão da jaqueta.

Apesar de muito promissora, a técnica ainda não está totalmente padronizada. Ainda não há consenso se ela é apropriada para lactentes prematuros [41-42] e já foi descrito que seus resultados podem diferir significantemente com pequenas alteraçôes na pressão de inflação e com a presença de suspiros [45,48]. Há também descrição da utilização deste método para avaliar e comprovar a eficácia técnicas de fisioterapia respiratória em lactentes [34].

\section{Realização do exame}

As PFP são procedimentos relativamente complexos que devem ser realizados por equipe treinada e experiente. Diversos fatores contribuem para sua melhor interpretação como a história clínica pregressa e atual, investigaçáo de antecedentes familiares e pessoais, particularmente dos neonatais e, investigação de fatores de risco. Exame físico completo, mensuração de dados antropométricos e sinais vitais também devem ser realizados no dia do exame. Recomenda-se que cada laboratório possua fichas padronizadas para coleta e armazenamento desses dados. Esses procedimentos simples são fundamentais para a identificação de possíveis fatores que contra-indicarão a PFP, como infecção aguda e doença do refluxo gastroesofágico. Atenção particular deve ser dada aos lactentes com história ou sinais de obstrução de via aérea alta que podem ser acentuados pela sedação [51]. As principais contraindicações das PFP estão listadas no quadro 1.

Apesar de alguns autores descreverem o estudo da função pulmonar em lactentes durante sono espontâneo, sem sedação, a grande maioria dos serviços envolvidos na sua avaliação emprega os derivados do Cloral como sedativo. Esses medicamentos são extremamente seguros, mas deve-se respeitar as contraindicaçôes como cardiopatias e neuropatias (quadro 1) [51]. Visando minimizar o risco de regurgitação e aspiração, a maioria dos centros experientes em PFP de lactentes recomenda jejum de pelo menos três ou quatro horas antes da realização da prova para evitar o refluxo gastroesofágico.

É fundamental que o paciente seja monitorizado durante todo o exame. O monitoramento clínico deve ser constante, observando-se a presença de sinais clínicos de desconforto respiratório e insuflaçáo gástrica. É realizado, também, o monitoramento contínuo da frequência cardíaca e da saturação de oxigênio. Fonte de oxigênio, sistema de aspiraçáo, material de reanimação e intubação devem estar disponíveis, além de equipe treinada para tanto.

O posicionamento do lactente pode influenciar os resultados da PFP, além de dificultar algumas medidas. Por isso, é importante que cada laboratório padronize a postura dos lactentes durante o exame. De modo geral, as provas de função pulmonar ainda são mais empregadas para pesquisa clínica [52], mas há grande avanço nas perspectivas da realização da mesma com outros objetivos como avaliação de técnicas de fisioterapia respiratória [34].

\section{Conclusão}

É consenso que a realização da PFP em adolescentes e crianças acima de seis anos faz parte da rotina de procedimentos para identificação de distúrbios respiratórios, observação de sua evolução e monitoramento do tratamento. Em lactentes, a avaliação da função pulmonar ainda é pouco divulgada, porém também pode auxiliar no diagnóstico e tratamento das pneumopatias em lactentes. 
Quadro 1 - Indicaçôes e contraindicaçôes das provas de função pulmonar em lactentes.

\begin{tabular}{ll}
\hline \multicolumn{1}{c}{ Indicações } & \multicolumn{1}{c}{ Contraindicações } \\
\hline Detectar a presença de doença pulmonar; & Refluxo gastroesofágico; \\
Avaliação e acompanhamento de crianças com doença obstrutiva ou restriti- & Neuropatias; \\
va crônica que não respondem ao tratamento clínico convencional; & Cardiopatias, especialmente as arrítmicas; \\
Definição da gravidade da doença respiratória e auxílio na terapia; & Obstrução de via aérea superior; \\
Avaliação de forma objetiva dos efeitos de terapias medicamentosas; & Cirurgias torácicas e/ou abdominais recentes. \\
Desenvolvimento de pesquisa científica. & \\
\hline
\end{tabular}

Os profissionais da saúde podem encontrar na PFP em lactentes elementos que auxiliarão a condução de sua terapia. O emprego desta ferramenta pode auxiliar a definiçáo diagnóstica, por vezes obscura, determinação de gravidade e assim nortear os objetivos e planejamento do tratamento. As PFP em lactentes também podem auxiliar na compreensão de padróes fisiológicos pulmonares bem como o acompanhamento de lactentes para observar a evolução de doenças. Assim, novas perspectivas a respeito da prova de função pulmonar em lactentes têm sido observadas e promissores resultados advindos da sua execução contribuirão cada vez mais na assistência ao lactente.

\section{Referências}

1. Burri PH. Fetal and postnatal development of the lung. ANNU Rev Physiol 1984; 46:617-628.

2. Thurlbeck WM. Postnatal human lung growth. Thorax 1982;37:564-71.

3. OPS/OMS. Las condiciones de salud en las Américas. Washington: Organização Pan-Americana da Saúde/Organização Mundial da Saúde 1994.

4. Glezen WP, Taber LH, Frank AL, Kasel JA. Risk of primary infection and reinfection with respiratory syncytial virus. A M J Dis Child 1986;140:543-6.

5. Chong Neto H, Rosário Filho N, Solé D, Mallol J. Prevalência de sibilância recorrente em lactentes. J Ped (Rio J) 2007;83:35762.

6. Dela Bianca A, Miyagi K, Camargo L, Cezarin D, Wandalsen G, Solé D. Estudo internacional de sibilâncias em lactentes (EISL): validação de questionário escrito para lactentes com até 36 meses de vida da cidade de São Paulo. Rev Bras Alerg Immunopatol 2007;30:232-9.

7. Tager IB, Hanrahan JP, Tosteson TD, Castile RG, Brown RW, Weiss ST, et al. Lung function, pre and post natal smoke exposure and wheezing in the first year of life. Am Rev Respir Dis 1993;147:811-7.

8. Wang X, Dockery DW, Wypoj D, Gold DR, Speizer FE, Ware $\mathrm{JH}$, et al. A longitudinal study of effects of parenatal smoking on pulmonary function in children 6-18 years. Am J Respir Crit Care Med 1994;149:1420-5.

9. Adler S, Wohl M. Flow-volume relationship at low lung volumes in healthy term newborn infants. Pediatrics 1978;61:636-40.

10. Stocks J, Sly P, Tepper R, Morgan W. Infant respiratory function testing. New York: Wiley Liss; 1996.

11. Grunstein MM, Springer C, Godfrey S, Bar-Yisahy E, Vilozni D, Inscore SC, et al. Expiratory volume clamping, a new method to asses respiratory mechanics in sedates infants. J Appl Physiol 1987;62:2107-14.

12. Jones M, Castile R, Davis S, Kisling J, Filbrun D, Flucke R, et al. Forced expiratory flows and volumes in infants - normative data and lung growth. Am J Respir Crit Care Med 2000;161:353-9.

13. Tepper RS, Morgan WJ, Cota K, Taussing LM, et al. Physiologic growth and development of the lung during the first year of life. Am Rev Respir Dis 1986;134:513-9.

14. Martinez FD, Wright AL, Taussing LM, Holberg CJ, Halomen M, Morgan WJ. Group health medical associates. Asthma and wheezing in the first six years of life. N Engl J Med 1995;332:133-8.

15. American Thoracic Society/European Respiratory Society. Statement: raised volume forced expirations in infants. Am J Respir Crit Care Med 2005;172:1463-71.

16. Milic-Emili J, Grundtein M. Drive and timing components of ventilation. Chest 1976;70:131-3.

17. Cutera R. Filtchev S, Merolla R, William G, Haluzka J, Ronchetti R. Analysis of expiratory pattern for monitoring bronchial obstruction in school age children. Pediatr Pulmonol 1991;10:6-10.

18. Kosch P, Davenport P, Wozniak J, Stark A. Control of inspiratory duration in newborn infants. J Appl Physol 1986;60:2007-14.

19. Kosch P, Davenport P, Wozniak J, Stark A. Control of expiratory duration in newborn infants. J Appl Physiol 1985;58:575-81.

20. Strick S, Ellis E, LeSouef P, Sly P. Validation of respiratory inductante plethysmography for the measurement of tidal breathing parameters in newborn. Pediatr Pulmonol 1992;14:187-91.

21. Martinez F, Morgan W, Wright A, Holberg C, Taussing L. Initial airway function is a risk for recurrent wheezing respiratory illnesses during first three years of life. Am Rev Respir Dis 1991;143:312-16.

22. Allen JL, Bar-Yishay E, Bryan AC, et al. Respiratory mechanics in infants: physiologic evaluations in health and disesae. Am Rev Resir Dis 1993;147:474-96.

23. Haland G, Carlsen K, Sandvik L, Devulapalli C, Kaas M, Petiersen $\mathrm{P}$, et al. Reduced lung function at birth and the risk of asthma at 10 years of age. N Engl J Med 2006;355:1682-9.

24. Rabbette PS, Costeloe KL, Stocks J. Persistence of the HeringBreuer reflex beyond the neonatal period. J Appl Physiol 1991;71:474-80.

25. LeSouef PN, England SJ, Brayan AC. Passive respiratory mechanics in newborn and children. Am Rev Respir Dis 1984;129:552-56.

26. Gappa M, Colin A, Goets I, Stocks J. Passive respiratory mechanics: the occlusion techniques. Eur Respir J 2001;17:141-8.

27. Mortola JP, Hemmings G, Matsuoka T, Saiki C, Fox G. Referencing lung volume for measurements of respiratory system compliance in infants. Pediatr Pulmonol 1993;16:248-53. 
28. Stocks J, Nothen U, Sutherland P, Hatch D, Helms P. Improved accuracy of the occlusion technique for assessing total respiratory compliance in infants. Pediatr Pulmonol 1987;3:71-7.

29. Rutter N, Milner AD, Hiller EJ. Effect of bronchodilators on respiratory resistance in infants and young children with bronchiolitis and wheezy bronchitis. Arch Dis Child 1975;50:71922.

30. Tepper RS, Pagtakhan RD, Taussing LM. Non invasive determination of total respiratory system compliance in infants by the weighted spirometer method. Am Rev Respir Dis 1984;130: 461-6.

31. Jarriel WS, Richardson P, Knapp RD, Hansen TN. A nonlinear regression analysis of nonlinear, passive-deflation flow-volume plots. Pediatr Pulmonol 1993;15:175-82.

32. Pfenniger J, Aebi C, BachmannD, Wagner BP. Lung mechanics and gas exchange in ventilated preterm infants during treatment of hyaline membrane disease with multiple doses of artificial surfactant. Pediatr Pulmonol 1992;14:10-15.

33. Brundage KL, Moshini KG, Froese AB, Walker CR, Fixher JT. Dexamethasone therapy for bronchopulmonary dysplasia: improved respiratory mechanics without adrenal suppression. Pediatr Pulmonol 1992;12:162-9.

34. Lanza FC. Técnica de fisioterapia respiratória expiração lenta e prolongada (ELPr): alterações funcionais pulmonares em lactentes sibilantes [Tese]. São Paulo: Universidade Federal de São Paulo / Escola Paulista de Medicina, 2009. 88f. Programa de Pós - Graduação em Pediatria.

35. DuBois AB, Botekho SY, Bedell GN, Marshall R, Cmroe JH. A rapid plethysmograph method for measuring TGV. J Clin Invest 1956;35:322-326.

36. Polgar G. Airway resistance in the newborn infant. J Pediatr 1961;59:915-21.

37. Auld PAM, Nelson NM, Cherry RB, Rudolph AJ, Smith CA. Measurement of thoracic gas volume in the newborn infant. J Clin Invest 1963;42:476-82.

38. Stocks J, Godfrey S, Beardsmore C, Bar-Yishay E, Castile R, ERS/ATS Task force on standards for infant respiratory function testing. Plethysmographic measurements of lung volume and airway resistance. Eur Respir J 2001;17:302-12.

39. Tepper RS, Hiatt P, Eigen H, Scott P, Grosfeld J, Cohen M. Infants with cystic fribosis: pulmonary function at diagnosis. Pediatr Pulmonol1988;5:15-8.
40. Sly P, Tepper R, Henschen M, Gappa M, Stocks J, ERS/ATS Task force on standards for infant respiratory function testing. Tidal forced expirations. Eur Respir J 2000;16:741-48.

41. Hulskamp G, Hoo A, Ljungberg H, Lum S, Pillow JJ, Stocks J. Progressive decline in plethysmographic lung volumes in infants physiology or technology? Am J Respir Crit Care Med 2003;168:1003-09.

42. Henschen M, Stocks J, Hoo A, Dixon P. Analysis of forced expiratory maneuvers from raised lung volume in preterm infants. J Appl Physiol 1998;1989-97.

43. Taussig L, Landau L, Godfrey S, Arad I. Determinants of forced expiratory flows in newborn infants. J Appl Physiol Respir Environ Exerc Physiol 1998;53:1220-7.

44. Martinez FD, Morgan WJ, Wright AL, Holgberg CJ, Taussing LM. Diminished lung function as a predisposing factor for wheezing respiratory illness in infants. $\mathrm{N}$ Engl J Med 1988;319:1112-7.

45. Lum S, Hoo A, Stocks J. Effect of airway inflation pressure on forced expiratory maneuvers from raised lung volume in infants. Pediatr Pulmonol 2002;33:130-4.

46. Modl M, Eber E, Weinhandl E, Gruber W, Zach MS. Reproducibility of forced expiratory flow and volume measurements in infants with bronchiolitis. Pediatr Pulmonol 1999;28:429-35.

47. Mallol J, Aguirre VL, Wandalsen G. Variability of the raised volume rapid thoracic compression technique in infants with recurrent wheezing. Allergol Immunopathol (Madr) 2005;33:74-9.

48. Wandalsen GF, La Scala CS, Lanza F, Molero Jr JC, Solé D. Influence of sights in the raised volume rapid thoracic compression technique (RVRTC) in infants. Pediatric Pulmonol 2008; 43:360-5.

49. Jones M, Howard J, Davis S, Kisiling J, Tepper R. Sensitivity of spirometric measurements to detect airway obstruction in infants. Am J Respir Crit Care Med 2003; 167:1283-6.

50. Turner D, Lanteri C, LeSouef P, Sly P. Improved detection of abnormal respiratory function using forced expiration from raised lung volume in infants with cystic fibrosis. Eur Resp J 1994;7:1995-99.

51. Leiter JC, Knuth SI, Bartlett D. The effect of sleep deprivation on activity of genioglossus muscles. Am Rev Resp Dis 1985;132:1242-5.

52. Godfrey S, Bar-Yishay E, Avital A, Springer C. What is the role of tests of lung function in the management of infants with lung disease? Pediatr Pulmonol 2003;36:1-9. 